Comment. Math. Helv. 78 (2003) 627-647

0010-2571/03/030627-21

DOI $10.1007 / \mathrm{s} 00014-003-0758-9$
(C) 2003 Birkhäuser Verlag, Basel

Commentarii Mathematici Helvetici

\title{
Fold maps on 4-manifolds
}

\author{
Osamu Saeki \\ Dedicated to Professor Mitsuyoshi Kato on his sixtieth birthday
}

\begin{abstract}
We give a complete characterization of those closed orientable 4-manifolds which admit smooth maps into $\mathbf{R}^{3}$ with only fold singularities. We also clarify the relationship between the existence problem of fold maps and that of linearly independent vector fields on manifolds.
\end{abstract}

Mathematics Subject Classification (2000). Primary 57R45; Secondary 57N13, 57R25.

Keywords. Fold map, 4-manifold, embedded surface, characteristic class, homotopy principle, vector field, stable span.

\section{Introduction}

Given a smooth map $f: M \rightarrow N$ between smooth manifolds $M$ and $N$ without boundary with $n=\operatorname{dim} M$ and $p=\operatorname{dim} N$, the following problem has been very important in differential topology: does there exist an immersion (for the case $n<p$ ) or a submersion (for the case $n \geq p$ ) of $M$ into $N$ homotopic to $f$ ? This problem is equivalent to finding a non-singular map in the homotopy class of a given map. In the literature, the target manifold $N$ is often taken to be the Euclidean space $\mathbf{R}^{p}$. For the immersion problem, this causes no problem: however, for the submersion problem, it does, since no compact manifold of dimension $n$ admits a submersion into $\mathbf{R}^{p}$ for $1 \leq p \leq n$. This observation leads us to consider smooth maps with as simplest singularities as possible instead of submersions, for the case $n \geq p$.

A singular point $q \in M$ of a smooth map $f: M \rightarrow N$ is of fold type if $f$ can be written in the form $\left(x_{1}, \ldots, x_{n}\right) \mapsto\left(x_{1}, \ldots, x_{p-1}, \pm x_{p}^{2} \pm \cdots \pm x_{n}^{2}\right)$ for some local coordinates around $q$ and $f(q)$. A smooth map $f$ is a fold map ${ }^{1}$ if all its singularities are of fold type. Singularities of fold type are the simplest among all generic singularities, so it is reasonable to consider fold maps instead of submersions when

The author has been supported in part by Grant-in-Aid for Scientific Research (No. 13640076), Ministry of Education, Science and Culture, Japan.

1 In [13], it is called a submersion with folds. 
the source manifold $M$ is compact and the target manifold $N$ is the $p$-dimensional Euclidean space $\mathbf{R}^{p}$ with $p \leq n=\operatorname{dim} M$.

As far as the author knows, the existence problem of fold maps goes back to Thom [39], who tried to generalize the theory of Morse functions to generic maps into the Euclidean spaces. Unfortunately, there have been very few studies on the existence problem of fold maps since Thom. Levine [20] has studied generic maps into $\mathbf{R}^{2}$ and has shown that a smooth closed manifold $M$ with $\operatorname{dim} M \geq 2$ admits a fold map into $\mathbf{R}^{2}$ if and only if its Euler characteristic is even. Èliašberg [10, 11] has systematically studied the existence problem of fold maps and obtained a socalled homotopy principle (in the existence level) for fold maps: i.e., any formal solution (in the 1-jet level) gives a genuine solution up to homotopy. However, his result was given in terms of $(p-1)$-dimensional submanifolds, and he did not give a characterization of manifolds admitting fold maps except for some special cases.

In [26], the author has shown that no 4-manifold $M$ that has the integral homology of $\mathbf{C} P^{2}$ admits a fold map into $\mathbf{R}^{3}$, by using Sakuma's modulo four formula [35], which is a consequence of Rohlin's result [25, 14] peculiar to 4dimensions. Such a phenomenon was not expected in view of Èliašberg's result and turned our attention to the problem again.

Recently, Ando [6] has obtained a very important result, which asserts that if there exists a fiberwise epimorphism $T M \oplus \varepsilon^{1} \rightarrow \varepsilon^{p}$, then $M$ admits a fold map into $\mathbf{R}^{p}$, where $T M$ and $\varepsilon^{k}$ denote the tangent bundle of $M$ and the trivial $k$-plane bundle over $M$ respectively. When $n-p+1$ is odd, the converse had already been known (for example, see [26]), so this gives a reasonable answer to the problem for half the cases.

In this paper, we consider the existence problem of fold maps for the case $(n, p)=(4,3)$, which is the first non-trivial case with $n-p+1$ being even. Our main result is the following complete answer to the problem.

Theorem 1.1. Let $M$ be a closed connected oriented 4-manifold. Then the following three are equivalent.

(1) There exists a fold map of $M$ into $\mathbf{R}^{3}$.

(2) The intersection form of $M$ is not isomorphic to

$$
\pm(1), \quad \pm\left(\begin{array}{ll}
1 & 0 \\
0 & 1
\end{array}\right) .
$$

(3) There exists a cohomology class $v \in H^{2}(M ; \mathbf{Z})$ such that $v \smile v=p_{1}(M) \in$ $H^{4}(M ; \mathbf{Z})$, where $p_{i}$ denotes the $i$-th Pontrjagin class.

The above result shows that there are a lot of 4-manifolds $M$ which admit fold maps into $\mathbf{R}^{3}$, but which have no fiberwise epimorphisms $T M \oplus \varepsilon^{1} \rightarrow \varepsilon^{3}$, or no fold maps into $\mathbf{R}^{2}$.

As is easily observed, for an $n$-dimensional manifold $M$, there exists a fiberwise epimorphism $T M \oplus \varepsilon^{1} \rightarrow \varepsilon^{p}$ if and only if there exist $p$ smooth sections of $T M \oplus \varepsilon^{1}$ which are everywhere linearly independent. Thus, the existence problem of fold 
maps is closely related to the (stable) vector field problem. In this paper, we also discuss their relationship.

The paper is organized as follows. In $\S 2$, we recall some basic properties of fold maps together with Ėliašberg's result [11], which plays a central role in this paper. In $\S 3$, we prove that the existence of certain embedded surfaces is equivalent to the existence of fold maps on 4-manifolds, by using Èliašberg's result. In $\S 4$, we study the existence of such embedded surfaces and complete the proof of our main theorem. In $\S 5$, we discuss the relationship between the fold map problem and the vector field problem.

Throughout the paper, all manifolds and maps are differentiable of class $C^{\infty}$. For a space $X, \operatorname{id}_{X}$ will denote the identity map of $X$.

The author would like to express his sincere gratitude to Kazuhiro Sakuma, Yakov Èliašberg, András Szücs, Peter Zvengrowski, and Julius Korbaš for stimulating discussions and invaluable comments.

\section{Preliminaries}

Let $f: M \rightarrow N$ be a smooth map between smooth manifolds with $n=\operatorname{dim} M \geq$ $\operatorname{dim} N=p$. A singular point of $f$ is a point $q \in M$ such that $\operatorname{rank} d f_{q}<p$. The set of all singular points of $f$ will be denoted by $S(f)$, which is called the singular set of $f$. A singular point $q \in M$ is of fold type if there exist local coordinates $\left(x_{1}, x_{2}, \ldots, x_{n}\right)$ and $\left(y_{1}, y_{2}, \ldots, y_{p}\right)$ around $q \in M$ and $f(q) \in N$ respectively such that

$$
y_{i} \circ f=\left\{\begin{array}{l}
x_{i}, \quad 1 \leq i \leq p-1, \\
-x_{p}^{2}-\cdots-x_{p+\lambda-1}^{2}+x_{p+\lambda}^{2}+\cdots+x_{n}^{2}, \quad i=p
\end{array}\right.
$$

for some integer $\lambda$ with $0 \leq \lambda \leq[(n-p+1) / 2]$, where [ $\ell]$ denotes the greatest integer not exceeding $\ell$. We call $\lambda$ the (reduced) index of $q$. We say that $f: M \rightarrow N$ is a fold map if all its singular points are of fold type.

Remark 2.1. Fold maps can also be characterized in terms of jets. Let $\Sigma_{r}$ denote the submanifold of the 1-jet bundle $J^{1}(M, N)$ consisting of the jets of corank $r$. Then a smooth map $f: M \rightarrow N$ is a fold map if and only if its 1-jet extension $j^{1} f: M \rightarrow J^{1}(M, N)$ does not hit $\Sigma_{2}$ and is transverse to $\Sigma_{1}$, and $f \mid\left(j^{1} f\right)^{-1}\left(\Sigma_{1}\right)$ is an immersion (for details, see [13, Chapter III, §4]). Fold singularities are the simplest, i.e. have the smallest codimension, among the generic singularities of corank one, i.e. among the Morin singularities [23].

For a fold map $f$ and $0 \leq \lambda \leq[(n-p+1) / 2]$, we denote by $S_{\lambda}(f)$ the set of all singular points of $f$ of fold type with index $\lambda$. It is easy to see that for a fold map $f$, each $S_{\lambda}(f)$ is always a $(p-1)$-dimensional regular closed submanifold of 
$M$, that $f \mid S_{\lambda}(f)$ is an immersion, and that we have the decomposition

$$
S(f)=\bigcup_{\lambda=0}^{[(n-p+1) / 2]} S_{\lambda}(f)
$$

(for details, see $[20,12]$ ).

For example, when $p=n-1$, we have exactly two possibilities for the reduced index $\lambda$; namely, $\lambda=0$ or 1 . In particular, when $(n, p)=(4,3)$, for a fold map $f: M \rightarrow N$, we have $S(f)=S_{0}(f) \cup S_{1}(f)$, where $S_{0}(f)$ and $S_{1}(f)$ are surfaces embedded in $M$. Sometimes we call $S_{0}(f)$ (or $S_{1}(f)$ ) the definite fold (resp. indefinite fold) of $f$.

Now let us recall Èliašberg's homotopy principle for fold maps. Let $M$ and $N$ be smooth manifolds of dimensions $n$ and $p$ respectively with $n \geq p$. The space of all fold maps of $M$ into $N$ is denoted by $\mathfrak{M}(M, N)$, which is endowed with the $C^{\infty}$ topology. Let $\Phi: T M \rightarrow T N$ be a homomorphism of vector bundles, where $T M$ and $T N$ denote the tangent bundles of $M$ and $N$ respectively. We say that $q \in M$ is a singular point of $\Phi$ if the rank of $\Phi_{q}: T_{q} M \rightarrow T_{\bar{\Phi}(q)} N$ is strictly less than $p$, where $\bar{\Phi}: M \rightarrow N$ is the continuous map induced by $\Phi$. By $\mathfrak{m}(M, N)$ we denote the space of all homomorphisms $\Phi: T M \rightarrow T N$ such that for each singular point $q \in M$, there exist a neighborhood $U \subset M$ of $q$ and a fold map $f_{U}: U \rightarrow N$ with $d f_{U}=\Phi \mid T U$. Note that $\mathfrak{m}(M, N)$ is endowed with the compact-open topology. For a singular point of a homomorphism $\Phi \in \mathfrak{m}(M, N)$, its (reduced) index is obviously defined. We denote the set of all singular points of $\Phi$ by $S(\Phi)$, and the set of all singular points of $\Phi$ of index $\lambda$ by $S_{\lambda}(\Phi), \lambda=0,1, \ldots,[(n-p+1) / 2]$.

Let $V_{0}, V_{1}, \ldots, V_{[(n-p+1) / 2]}$ be disjoint $(p-1)$-dimensional regular closed submanifolds of $M$. We denote by $\mathfrak{M}\left(M, N ; V_{0}, V_{1}, \ldots, V_{[(n-p+1) / 2]}\right.$ ) (or by $\left.\mathfrak{m}\left(M, N ; V_{0}, V_{1}, \ldots, V_{[(n-p+1) / 2]}\right)\right)$ the subspace of $\mathfrak{M}(M, N) \quad(\operatorname{resp} \cdot \mathfrak{m}(M, N))$ formed by the smooth maps $f: M \rightarrow N$ with $S_{\lambda}(f)=V_{\lambda}$ (resp. by the homomorphisms $\Phi: T M \rightarrow T N$ with $\left.S_{\lambda}(\Phi)=V_{\lambda}\right)$ for $\lambda=0,1, \ldots,[(n-p+1) / 2]$.

We have the natural map

$$
d: \mathfrak{M}\left(M, N ; V_{0}, V_{1}, \ldots, V_{[(n-p+1) / 2]}\right) \rightarrow \mathfrak{m}\left(M, N ; V_{0}, V_{1}, \ldots, V_{[(n-p+1) / 2]}\right)
$$

defined by $d(f)=d f: T M \rightarrow T N$. The following theorem has been proved by Èliašberg [11] and will play an essential role in this paper.

Theorem 2.2. If $M$ is connected, $p \geq 2$, and the $(p-1)$-dimensional submanifolds $V_{\lambda}$ are all non-empty for $\lambda=0,1, \ldots,[(n-p+1) / 2]$, then the map

$d_{*}: \pi_{0}\left(\mathfrak{M}\left(M, N ; V_{0}, V_{1}, \ldots, V_{[(n-p+1) / 2]}\right)\right) \rightarrow \pi_{0}\left(\mathfrak{m}\left(M, N ; V_{0}, V_{1}, \ldots, V_{[(n-p+1) / 2]}\right)\right)$ is surjective.

Remark 2.3. Let us consider the following problem: given a collection of disjoint $(p-1)$-dimensional closed submanifolds of $M$, does there exist a fold map into $N$ which realizes them as its singular set (respecting the indices)? An element 
of $\mathfrak{m}\left(M, N ; V_{0}, V_{1}, \ldots, V_{[(n-p+1) / 2]}\right)$ can be regarded as a formal solution to this problem. Then the above theorem asserts that any formal solution gives us a real solution up to homotopy, provided that all the indices appear. This last condition is essential. For example, the 4-dimensional torus $T^{4}$ is parallelizable, so that it admits a formal solution for some 2-dimensional submanifold $V_{0}$, where $N=\mathbf{R}^{3}$. However, $T^{4}$ does not admit a fold map into $\mathbf{R}^{3}$ without indefinite folds (see $[31,33])$.

\section{Fold maps and embedded surfaces}

In this section, we characterize those surfaces embedded in a 4-manifold such that the corresponding spaces of formal solutions are non-empty.

In the following, $\chi$ will denote the Euler characteristic. The purpose of this section is to prove the following.

Theorem 3.1. Let $M$ be a closed connected oriented 4-manifold and $F$ a closed surface embedded in $M$. Suppose that $F$ decomposes into a disjoint union $F=$ $F_{0} \cup F_{1}$, where $F_{0}$ and $F_{1}$ are non-empty and consist of some connected components of $F$. Then there exists a fold map $f: M \rightarrow \mathbf{R}^{3}$ with $S_{0}(f)=F_{0}$ and $S_{1}(f)=F_{1}$ if and only if the following five conditions hold.

(1) $\chi(M)=\chi\left(F_{0}\right)-\chi\left(F_{1}\right)$.

(2) The embedded surface $F$ is characteristic; i.e., the $\mathbf{Z} / 2 \mathbf{Z}$-homology class $[F]_{2} \in H_{2}(M ; \mathbf{Z} / 2 \mathbf{Z})$ represented by $F$ is Poincaré dual to the second StiefelWhitney class $w_{2}(M) \in H^{2}(M ; \mathbf{Z} / 2 \mathbf{Z})$.

(3) The surface $F_{0}$ is orientable.

(4) The self-intersection number $F_{1}^{j} \cdot F_{1}^{j}$ in $M$ vanishes for each connected component $F_{1}^{j}$ of $F_{1}$.

(5) The self-intersection number $F \cdot F=\left(F_{0} \cdot F_{0}\right)+\left(F_{1} \cdot F_{1}\right)$ coincides with $\left\langle p_{1}(M),[M]\right\rangle$, where $p_{1}(M) \in H^{4}(M ; \mathbf{Z})$ denotes the first Pontrjagin class, $[M] \in H_{4}(M ; \mathbf{Z})$ denotes the fundamental class of $M$, and $\langle$,$\rangle denotes the$ Kronecker product.

Proof. Suppose that there exists a fold map $f: M \rightarrow \mathbf{R}^{3}$. Then $S(f)$ decomposes into $F_{0}=S_{0}(f)$ and $F_{1}=S_{1}(f)$. From Fukuda [12], (1) follows (see also [28]). Furthermore, (2) follows from Thom [39]. Item (3) follows from the fact that the normal bundle of the immersion $f \mid S_{0}(f)$ is always trivial (see, for example, [26]). Item (4) follows from [26, Corollary 2.7]. Finally, (5) follows from [24]. In fact, (5) follows also from the argument below (for details, see Remark 3.6).

Suppose, conversely, that an embedded surface $F=F_{0} \cup F_{1}$ satisfying the above properties (1)-(4) is given. Note that, for the moment, we do not assume condition (5).

Let $N(F)=N\left(F_{0}\right) \cup N\left(F_{1}\right)$ be a closed tubular neighborhood of $F$, where 
$N\left(F_{i}\right)$ is a tubular neighborhood of $F_{i}, i=0,1$. Since $F_{0}$ is orientable, $N\left(F_{0}\right)$ is diffeomorphic to a $D^{2}$-bundle over $F_{0}$ with structure group $S O(2)$, where $D^{2}$ is the unit disk in $\mathbf{R}^{2}$. Using the $S O(2)$-invariant function $\alpha: D^{2} \rightarrow[0,1]$ defined by $\alpha(x, y)=x^{2}+y^{2}$ on each fiber, we obtain a fold map $\varphi_{0}: N\left(F_{0}\right) \rightarrow F_{0} \times[0,1]$ such that $S\left(\varphi_{0}\right)=S_{0}\left(\varphi_{0}\right)=F_{0}$. Then, for an arbitrary immersion $\eta_{0}: F_{0} \times[0,1] \uparrow \mathbf{R}^{3}$, the smooth map $f_{0}=\eta_{0} \circ \varphi_{0}: N\left(F_{0}\right) \rightarrow \mathbf{R}^{3}$ is a fold map such that $S\left(f_{0}\right)=$ $S_{0}\left(f_{0}\right)=F_{0}$.

Let $F_{1}=F_{1}^{1} \cup F_{1}^{2} \cup \cdots \cup F_{1}^{\ell}$ be the decomposition into the connected components of $F_{1}$. Note that $N\left(F_{1}\right)$ decomposes into the union of the tubular neighborhoods $N\left(F_{1}^{j}\right)$ of $F_{1}^{j}, j=1,2, \ldots, \ell$. By our assumption (4), if $F_{1}^{j}$ is orientable, then $N\left(F_{1}^{j}\right)$ is diffeomorphic to $F_{1}^{j} \times D^{2}$. Let $\beta: D^{2} \rightarrow[-1,1]$ be the function defined by $\beta(x, y)=x^{2}-y^{2}$. Then, for an arbitrary immersion $\eta_{1}^{j}: F_{1}^{j} \times[-1,1] \leftrightarrow \mathbf{R}^{3}$, the smooth map $f_{1}^{j}=\eta_{1}^{j} \circ\left(\operatorname{id}_{F_{1}^{j}} \times \beta\right): N\left(F_{1}^{j}\right) \cong F_{1}^{j} \times D^{2} \rightarrow \mathbf{R}^{3}$ is a fold map such that $S\left(f_{1}^{j}\right)=S_{1}\left(f_{1}^{j}\right)=F_{1}^{j}$.

On the other hand, when $F_{1}^{j}$ is non-orientable, let $\widetilde{F}_{1}^{j}$ be its orientation double cover and $\tau: \widetilde{F}_{1}^{j} \rightarrow \widetilde{F}_{1}^{j}$ the covering translation. Then, our assumption (4) implies that $N\left(F_{1}^{j}\right)$ is diffeomorphic to $F_{1}^{j} \widetilde{\times} D^{2}=\left(\widetilde{F}_{1}^{j} \times D^{2}\right) /(\tau \times \rho)$, where $\rho: D^{2} \rightarrow D^{2}$ is the reflection defined by $\rho(x, y)=(y, x)$. Let $F_{1}^{j} \widetilde{\times} I=\left(\widetilde{F}_{1}^{j} \times I\right) /(\tau \times r)$ be the twisted $I$-bundle over $F_{1}^{j}$, where $I=[-1,1]$ and $r: I \rightarrow I$ is the reflection defined by $r(t)=-t$. Then the diagram

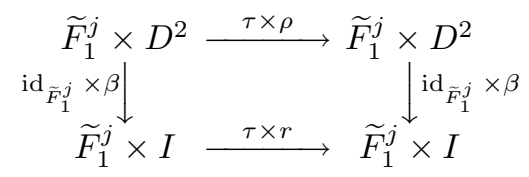

is commutative. Thus $\operatorname{id}_{\widetilde{F}_{1}^{j}} \times \beta$ induces the fold map $\varphi_{1}^{j}: N\left(F_{1}^{j}\right) \cong F_{1}^{j} \widetilde{\times} D^{2} \rightarrow$ $F_{1}^{j} \tilde{\times} I$. Then, for an arbitrary immersion $\eta_{1}^{j}: F_{1}^{j} \widetilde{\times} I \leftrightarrow \mathbf{R}^{3}$, the map $f_{1}^{j}=\eta_{1}^{j}$ 。 $\varphi_{1}^{j}: N\left(F_{1}^{j}\right) \rightarrow \mathbf{R}^{3}$ is a fold map such that $S\left(f_{1}^{j}\right)=S_{1}\left(f_{1}^{j}\right)=F_{1}^{j}$. Note that such an immersion $\eta_{1}^{j}$ does exist, since $F_{1}^{j} \widetilde{\times} I$ is an orientable 3 -manifold and so is parallelizable.

Thus, we have constructed a fold map $f_{N(F)}=f_{0} \cup\left(f_{1}^{1} \cup \cdots \cup f_{1}^{\ell}\right): N(F) \rightarrow \mathbf{R}^{3}$, and hence a homomorphism $T N(F) \rightarrow T \mathbf{R}^{3}$. In order to use Theorem 2.2, we would like to extend this homomorphism through $T M$ so that it is non-singular on the complement of $N(F)$.

Note that $f_{N(F)} \mid(N(F) \backslash F): N(F) \backslash F \rightarrow \mathbf{R}^{3}$ is a submersion. Hence, we have the nowhere vanishing vector fields $\xi_{0}, \xi_{1}, \xi_{2}$ and $\xi_{3}$ on $N(F) \backslash F$ such that

$$
d f_{N(F)}\left(\xi_{i, q}\right)=\left(\frac{\partial}{\partial x_{i}}\right)_{f_{N(F)}(q)}
$$

for all $q \in N(F) \backslash F$ and $i=1,2,3$, and that $\xi_{0}$ is tangent to the fibers of $f_{N(F)}$, where $\left(x_{1}, x_{2}, x_{3}\right)$ are the usual coordinates of $\mathbf{R}^{3}$. Furthermore, we may assume that the ordered 4 -tuple $\left(\xi_{0}, \xi_{1}, \xi_{2}, \xi_{3}\right)$ is coherent with the orientation of $M$. By 
Theorem 2.2, in order to construct a desired fold map on $M$, we have only to extend $\xi_{0}, \xi_{1}, \xi_{2}$ and $\xi_{3}$ to nowhere linearly dependent vector fields on $M \backslash F$.

Set $X=M \backslash \operatorname{Int} N(F)$ and let $\phi$ denote the framing of $T X$ over $\partial X=\partial N(F)$ determined by $\left(\xi_{0}, \xi_{1}, \xi_{2}, \xi_{3}\right)$. Note that $\phi$ can be regarded as a stable framing of the oriented 3-manifold $\partial X$. Let $d(\phi) \in \mathbf{Z}$ denote its degree (for details, see [18]).

Let $F_{0}=F_{0}^{1} \cup \cdots \cup F_{0}^{k}$ and $F_{1}=F_{1}^{1} \cup \cdots \cup F_{1}^{\ell}$ be the decompositions into the connected components and denote $\overline{\phi \mid E_{i}^{j}}$ by $\phi_{i}^{j}$, where $E_{i}^{j}$ denotes the boundary of the closed tubular neighborhood $N\left(F_{i}^{j}\right)$ of $F_{i}^{j}$ and is oriented as the boundary of $N\left(F_{i}^{j}\right)$, and $\overline{\phi \mid E_{i}^{j}}$ denotes the stable framing of $E_{i}^{j}$ obtained by negating the last vector field of $\phi \mid E_{i}^{j}$.

Lemma 3.2. For the degree of the stable framing $\phi_{i}^{j}$, we have

$$
d\left(\phi_{i}^{j}\right)= \begin{cases}0, & i=0, \\ -2 \chi\left(F_{1}^{j}\right), & i=1 .\end{cases}
$$

Proof. Recall that the degree of the stable framing $\phi_{i}^{j}$ is defined to be the degree of the map $\nu_{i}^{j}: E_{i}^{j} \rightarrow S^{3}$ which assigns to each point of $E_{i}^{j}$ the position of the outward normal in the 3 -sphere determined by $\phi_{i}^{j}$.

For $\phi_{0}^{j}$, one of its four vector fields is tangent to the 3 -manifold $E_{0}^{j}$. Thus, $\nu_{0}^{j}$ is a map into a 2-sphere embedded in $S^{3}$, and hence $d\left(\phi_{0}^{j}\right)=0$. For $\phi_{1}^{j}$, the contribution to $d\left(\phi_{1}^{j}\right)$ from the base space $F_{1}^{j}$ of $E_{1}^{j}$ is equal to the Euler characteristic $\chi\left(F_{1}^{j}\right)$ and that from a fiber is equal to -2 by the construction of our vector fields. Hence, we have $d\left(\phi_{1}^{j}\right)=-2 \chi\left(F_{1}^{j}\right)$.

By the above lemma together with the additivity of the degree, we have

$$
d(\phi)=-2 \chi\left(F_{1}\right)=\left(\chi\left(F_{0}\right)-\chi\left(F_{1}\right)\right)-\chi(F)=\chi(M)-\chi(N(F))=\chi(X),
$$

where the third equality follows from our assumption (1) (see [18, Theorem 2.2] for the property of the degree with respect to the orientation reversal).

Remark 3.3. We have an alternative proof for the equality $d(\phi)=\chi(X)$ as follows. By the construction of the vector field $\xi_{0}$, it winds once around $F_{0}$ on each fiber of $N\left(F_{0}\right) \backslash F_{0} \rightarrow F_{0}$ : i.e., it has index +1 along $F_{0}$ in the sense of [8]. Furthermore, it has index -1 along $F_{1}$. Hence, by a generalized Poincaré-Hopf theorem (see [8, Theorem 3.2]) together with our condition (1), we see that $\xi_{0}$ extends through $X$ as a nowhere vanishing vector field. Hence $d(\phi)=\chi(X)$ follows.

By our condition (2), $M \backslash F$ is a spin manifold: i.e., for a triangulation of $M$, there exists a trivialization of $T M$ over $M^{(2)} \backslash F$, where $M^{(i)}$ denotes the $i$-skeleton of $M$. To be more precise, let us take a triangulation of $M$ such that $M^{(1)} \cap F=\emptyset$ and that every 2-simplex $\sigma$ with $\sigma \cap F \neq \emptyset$ is contained in $N(F)$ and intersects $F$ transversely at a unique point in its interior. Then, there exists a trivialization 
of $T M$ over $M^{(2)} \backslash F$ such that for each 2-simplex $\sigma$ of $M$ with $\sigma \cap F \neq \emptyset$, the trivialization over $\partial \sigma$ corresponds to the generator of $\pi_{1}(S O(4)) \cong \mathbf{Z} / 2 \mathbf{Z}$ when compared with a trivialization which extends through $\sigma$. On the other hand, the ordered 4-tuple of vector fields $\left(\xi_{0}, \xi_{1}, \xi_{2}, \xi_{3}\right)$ gives a trivialization of $T M$ over $M^{(2)} \cap(N(F) \backslash F)$. By the construction, we see that for each 2-simplex $\sigma$ of $M$ with $\sigma \cap F \neq \emptyset$, this also corresponds to the generator of $\pi_{1}(S O(4))$. This means that the stable framing $\phi$ over $\partial X$ is compatible with a spin structure on $X$.

Then, in the above situation, the stable framing $\phi$ on $\partial X$ extends through $X$ if and only if the relative Pontrjagin number $p_{1}(X, \phi) \in H^{4}(X, \partial X ; \mathbf{Z}) \cong \mathbf{Z}$ vanishes (for details, see [18, Lemma 2.3]). Hence, in order to prove our theorem, we have only to prove

$$
p_{1}(X, \phi)=\left\langle p_{1}(M),[M]\right\rangle-F \cdot F
$$

Note that $\partial X$ is oriented as the boundary of $X$.

Let $h(\phi) \in \mathbf{Z}$ denote the Hirzebruch defect of the stable framing $\phi$ of $\partial X$ (for details, see [18]). In our situation, we have $h(\phi)=p_{1}(X, \phi)-3 \sigma(X)$, where $\sigma(X)$ denotes the signature of the oriented 4-manifold $X$. (Recall that $h(\phi)$ depends only on the stable framing $\phi$ on $\partial X$ and not on a particular choice of an oriented 4-manifold bounded by $\partial X$.) By the additivity of the Hirzebruch defect, we have

$$
h(\phi)=-h\left(\phi_{0}^{1}\right)-\cdots-h\left(\phi_{0}^{k}\right)-h\left(\phi_{1}^{1}\right)-\cdots-h\left(\phi_{1}^{\ell}\right)
$$

(see [18, Theorem 2.5] for the property of the Hirzebruch defect with respect to the orientation reversal).

Lemma 3.4. Let $e_{0}^{j} \in \mathbf{Z}$ denote the Euler number of the oriented $D^{2}$-bundle $N\left(F_{0}^{j}\right) \rightarrow F_{0}^{j}$. Then we have the following.

$$
h\left(\phi_{0}^{j}\right)= \begin{cases}0, & \text { if } e_{0}^{j}=0, \\ e_{0}^{j}-3, & \text { if } e_{0}^{j}>0, \\ e_{0}^{j}+3, & \text { if } e_{0}^{j}<0 .\end{cases}
$$

(2) $h\left(\phi_{1}^{j}\right)=0$ for all $j$.

Proof. Let $\pi: E \rightarrow \Sigma$ be the oriented $S^{1}$-bundle of Euler number $e \in \mathbf{Z}$ over a closed connected oriented surface $\Sigma$. Let us consider an immersion $\eta: \Sigma \rightarrow \mathbf{R}^{3}$. Since its normal bundle is trivial, by pulling back the standard framing of $\mathbf{R}^{3}$ by $\eta \circ \pi$, we obtain a stable framing of $E, \phi=\left(\xi_{0}, \xi_{1}, \xi_{2}, \xi_{3}\right)$, such that $\xi_{0}$ is tangent to the fibers of $\pi$. Let $\widetilde{\pi}: \widetilde{E} \rightarrow \Sigma$ be the oriented $D^{2}$-bundle associated with the oriented $S^{1}$-bundle $\pi: E \rightarrow \Sigma$. In order to prove Lemma 3.4 (1), we have only to prove that $p_{1}(\widetilde{E}, \phi)=e$.

By the very definition, we have

$$
p_{1}(\widetilde{E}, \phi)=-c_{2}\left((T \widetilde{E})_{\mathbf{C}}, \phi_{\mathbf{C}}\right) \in H^{4}\left(\widetilde{E}, E ;\left\{\pi_{3}(U(4) / U(1))\right\}\right) \cong \mathbf{Z},
$$


where $(T \widetilde{E})_{\mathbf{C}}$ is the complexification of the real tangent bundle $T \widetilde{E}$ of $\widetilde{E}, \phi_{\mathbf{C}}$ is the complexification of the 3-field $\left(\xi_{1}, \xi_{2}, \xi_{3}\right)$ over the boundary $\partial \widetilde{E}=E$, and $c_{2}$ denotes the second Chern class which coincides with the obstruction to finding a complex 3-field on $(T \widetilde{E})_{\mathbf{C}}$ over $\widetilde{E}$ extending $\phi_{\mathbf{C}}$. Note that we have canonically $\pi_{3}(U(4) / U(1)) \cong \pi_{3}(S U(4)) \cong \mathbf{Z}$ (for details, see [18]).

Let $\Sigma=h^{0} \cup\left(h_{1}^{1} \cup \cdots \cup h_{2 g}^{1}\right) \cup h^{2}$ be the standard handle decomposition of the closed connected oriented surface $\Sigma$, where $h^{0}, h_{j}^{1}$ and $h^{2}$ are 0-, 1- and 2-handles respectively, and $g$ is the genus of $\Sigma$. Since $\widetilde{\pi}: \widetilde{E} \rightarrow \Sigma$ is trivial over each of the above handles, we have the decomposition $\widetilde{E}=\widetilde{h}^{0} \cup\left(\widetilde{h}_{1}^{1} \cup \cdots \cup \widetilde{h}_{2 g}^{1}\right) \cup \widetilde{h}^{2}$, where $\widetilde{h}^{0}=D^{2} \times h^{0}, \widetilde{h}_{j}^{1}=D^{2} \times h_{j}^{1}$ and $\widetilde{h}^{2}=D^{2} \times h^{2}$ are 0 -, 1- and 2-handles respectively. Taking the dual decomposition, we have $\widetilde{E}=E \cup \widehat{h}^{2} \cup\left(\widehat{h}_{1}^{3} \cup \cdots \cup \widehat{h}_{2 g}^{3}\right) \cup \widehat{h}^{4}$, where $\widehat{h}^{2}, \widehat{h}_{j}^{3}$ and $\widehat{h}^{4}$ are the dual 2-, 3- and 4-handles respectively. Since $\pi_{i}(U(4) / U(1))=$ 0 for $i=1,2$, we can extend the 3 -field $\phi_{\mathbf{C}}$ over $E \cup \widehat{h}^{2} \cup\left(\widehat{h}_{1}^{3} \cup \cdots \cup \widehat{h}_{2 g}^{3}\right)$. Let us continue to denote the extended vector fields by $\xi_{1}, \xi_{2}$ and $\xi_{3}$ respectively. Note that then $\xi_{0}$ can also be extended so that $\left(\xi_{0}, \xi_{1}, \xi_{2}, \xi_{3}\right)$ gives a section of the associated $S U(4)$-bundle.

Let us consider a trivialization of $(T \widetilde{E})_{\mathbf{C}}$ over $\widehat{h}^{4}=\widetilde{\pi}^{-1}\left(h^{0}\right)$ which is compatible with a trivialization $\widetilde{\pi}^{-1}\left(h^{0}\right) \cong D^{2} \times h^{0}$ : more precisely, we take a trivialization which respects the isomorphism $(T \widetilde{E})_{\mathbf{C}} \mid \widetilde{\pi}^{-1}\left(h^{0}\right) \cong p_{1}^{*}\left(T D^{2}\right)_{\mathbf{C}} \oplus p_{2}^{*}\left(T h^{0}\right)_{\mathbf{C}}$, where $p_{1}: D^{2} \times h^{0} \rightarrow D^{2}$ and $p_{2}: D^{2} \times h^{0} \rightarrow h^{0}$ are the projections. Since $\pi_{i}(S U(4) / S U(2))=0$ for $i \leq 3$, we may assume that $\left(\xi_{2}, \xi_{3}\right)$ gives the trivialization of $p_{2}^{*}\left(T h^{0}\right)_{\mathbf{C}}$ over the boundary of $\widehat{h}^{4}$ by changing $\phi$ by a homotopy. We may further assume that $\left(\xi_{0}, \xi_{1}\right)$ on $\pi^{-1}\left(h^{0}\right) \cong S^{1} \times h^{0}$ corresponds to the map

$$
S^{1} \times h^{0} \stackrel{p_{1}^{\prime}}{\longrightarrow} S^{1}=S O(2) \stackrel{R_{L}}{\longrightarrow} S O(2) \hookrightarrow S U(2)(\subset S U(4))
$$

with respect to our trivialization, where $p_{1}^{\prime}$ is the projection to the first factor and $R_{L}$ is the right multiplication by the matrix

$$
L=\left(\begin{array}{rr}
0 & -1 \\
1 & 0
\end{array}\right) \in S O(2)
$$

In particular, $\left(\xi_{0}, \xi_{1}\right)$ is $S O(2)$-equivariant in the sense that

$$
\left(\xi_{0}(u v, x), \xi_{1}(u v, x)\right)=\left(\xi_{0}(u, x), \xi_{1}(u, x)\right) v
$$

holds for all $x \in h^{0}$ and $u, v \in S^{1}=S O(2) \subset S U(2)$.

Let $\varphi$ be the attaching map of the dual 4-handle $\widehat{h}^{4}$. Recall that $p_{1}(\widetilde{E}, \phi)$ coincides with -1 times the obstruction in question by (3.3). Hence, in order to prove Lemma $3.4(1)$, we have only to show that the framing

$$
\left(\widehat{\xi}_{0}, \widehat{\xi}_{1}, \widehat{\xi}_{2}, \widehat{\xi}_{3}\right)=\left(\varphi^{*}\left(\xi_{0}\right), \varphi^{*}\left(\xi_{1}\right), \varphi^{*}\left(\xi_{2}\right), \varphi^{*}\left(\xi_{3}\right)\right)
$$

over $\partial \widehat{h}^{4}$ corresponds to $-e \in \mathbf{Z}$ with respect to the natural isomorphism

$$
\pi_{3}(S U(4)) \cong \mathbf{Z}
$$


where we use our trivialization of $(T \widetilde{E})_{\mathbf{C}}$ over $\widehat{h}^{4}$ for identifying the framing (3.4) with a map into $S U(4)$. Note that $\partial \widehat{h}^{4}=\pi^{-1}\left(h^{0}\right) \cup \widetilde{\pi}^{-1}\left(\partial h^{0}\right)=\left(S^{1} \times h^{0}\right) \cup\left(D^{2} \times\right.$ $\left.\partial h^{0}\right)$ and $\varphi(u, x)=\left(u x^{-e}, x\right)$ for all $(u, x) \in D^{2} \times \partial h^{0}$, where we identify $D^{2}$ with the unit disk in $\mathbf{C}$, and $\partial h^{0}$ with the unit circle in $\mathbf{C}$.

By the above construction, $\left(\widehat{\xi}_{0}, \widehat{\xi}_{1}, \widehat{\xi}_{2}, \widehat{\xi}_{3}\right)=\left(\varphi^{*}\left(\xi_{0}\right), \varphi^{*}\left(\xi_{1}\right), \varphi^{*}\left(\xi_{2}\right), \varphi^{*}\left(\xi_{3}\right)\right)$ satisfies $\left(\widehat{\xi}_{0}(u, x), \widehat{\xi}_{1}(u, x), \widehat{\xi}_{2}(u, x), \widehat{\xi}_{3}(u, x)\right)=u L \oplus(1) \oplus(1)$ for all $(u, x) \in S^{1} \times h^{0}$, where we identify $S^{1}$ with $S O(2) \subset S U(2)$. Then $\left(\widehat{\xi}_{0}, \widehat{\xi}_{1}, \widehat{\xi}_{2}, \widehat{\xi}_{3}\right)$ over $D^{2} \times \partial h^{0}$ induces a map $\Phi: D^{2} \times S^{1} \rightarrow S U(4)$ such that $\Phi(u, x)=u L \oplus(1) \oplus$ (1) for all $u \in \partial D^{2}=S^{1}=S O(2) \subset S U(2)$ and $x \in S^{1}$. Thus, $\Phi$ induces a map $\bar{\Phi}:\left(D^{2} \times S^{1}\right) / \sim=S^{3} \rightarrow S U(4)$, where we define $(u, x) \sim\left(u^{\prime}, x^{\prime}\right)$ for $(u, x),\left(u^{\prime}, x^{\prime}\right) \in D^{2} \times S^{1}$ if and only if they lie on $\partial D^{2} \times S^{1}$ and $u=u^{\prime}$. We have only to show that $\bar{\Phi}$ corresponds to $-e \in \mathbf{Z}$ with respect to the natural isomorphism $\pi_{3}(S U(4)) \cong \mathbf{Z}$.

Since $\pi_{3}(S U(4))$ is abelian, the contribution from that part of $\partial h^{0}$ where the 1-handles $h_{j}^{1}$ are attached is zero. Thus we have only to consider the contribution from the "twist" by $e$. Since $\pi_{2}(S U(4))$ vanishes, we see that the homotopy class of $\bar{\Phi}$ does not depend on the choice of the map $\Phi \mid\left(D^{2} \times 1\right), 1 \in S^{1} \subset \mathbf{C}$, or equivalently, on the extension of $\left(\xi_{0}, \xi_{1}, \xi_{2}, \xi_{3}\right)$ over the fiber $\widetilde{\pi}^{-1}\left(x_{0}\right)$ for a point $x_{0} \in \partial h^{0}$. Hence we may assume that

$$
\Phi(r \exp (\sqrt{-1} \theta), 1)=\left(\begin{array}{cc}
u(r, \theta) & -\bar{v}(r, \theta) \\
v(r, \theta) & \bar{u}(r, \theta)
\end{array}\right) \oplus I_{2} \in S U(4),
$$

where $0 \leq r \leq 1,0 \leq \theta \leq 2 \pi$,

$$
\begin{array}{ll}
u(r, \theta)=-r \sin \theta-\sqrt{-1} \sqrt{1-r^{2}}, & \bar{u}(r, \theta)=-r \sin \theta+\sqrt{-1} \sqrt{1-r^{2}}, \\
v(r, \theta)=r \cos \theta, & \bar{v}(r, \theta)=r \cos \theta,
\end{array}
$$

and $I_{2}$ is the $2 \times 2$ identity matrix. Note that $\Phi(r \exp (\sqrt{-1} \theta), 1)$ does not depend on $\theta$ for $r=0$ and hence it is well-defined. Then for $\tau \in[0,2 \pi]$, we have

$$
\begin{aligned}
& \Phi(r \exp (\sqrt{-1} \theta), \exp (\sqrt{-1} \tau)) \\
= & \left(\begin{array}{cc}
u(r, \theta-e \tau) & -\bar{v}(r, \theta-e \tau) \\
v(r, \theta-e \tau) & \bar{u}(r, \theta-e \tau)
\end{array}\right)\left(\begin{array}{cc}
\cos (e \tau) & -\sin (e \tau) \\
\sin (e \tau) & \cos (e \tau)
\end{array}\right) \oplus I_{2} \\
= & \left(\begin{array}{cc}
-r \sin \theta-\sqrt{-1}(\cos e \tau) \sqrt{1-r^{2}} & -r \cos \theta+\sqrt{-1}(\sin e \tau) \sqrt{1-r^{2}} \\
r \cos \theta+\sqrt{-1}(\sin e \tau) \sqrt{1-r^{2}} & -r \sin \theta+\sqrt{-1}(\cos e \tau) \sqrt{1-r^{2}}
\end{array}\right) \oplus I_{2} .
\end{aligned}
$$

Then it is not difficult to see that the map $D^{2} \times S^{1} / \sim \rightarrow S^{3}$ defined by the first column vector of $\Phi$,

$$
(r \exp (\sqrt{-1} \theta), \exp (\sqrt{-1} \tau)) \mapsto\left(\begin{array}{c}
-r \sin \theta-\sqrt{-1}(\cos e \tau) \sqrt{1-r^{2}} \\
r \cos \theta+\sqrt{-1}(\sin e \tau) \sqrt{1-r^{2}} \\
0 \\
0
\end{array}\right) \in \mathbf{C}^{2} \times\{0\},
$$

has degree $e$, where we identify $S^{3}$ with the unit sphere of $\mathbf{C}^{2}$. Hence $\bar{\Phi}$ corresponds 
to $-e$ times the natural generator of $\pi_{3}(S U(4))$ (for the sign, see Remark 3.5 below). This completes the proof of Lemma 3.4 (1).

Remark 3.5. As explained carefully in $[18, \S 1]$, the natural generator $\sigma$ of

$$
\pi_{3}(S U(2)) \cong \mathbf{Z}
$$

is constructed by using an identification of $\mathbf{C}^{2}$ with the quaternions $\mathbf{H}$ as follows: $\sigma(q)=q x$, for $q \in S^{3} \subset \mathbf{H}$ and $x \in \mathbf{C}^{2}=\mathbf{H}$. However, for $\sigma$ to induce a unitary action, the vector $\left(x_{1}, x_{2}\right) \in \mathbf{C}^{2}$ must be identified with the quaternion $u+j v$ rather than $u+v j$. Hence, the map $S^{3} \rightarrow S^{3}$ defined by the first column vector of $\sigma$ has degree -1 .

Let us now prove Lemma $3.4(2)$. When $F_{1}^{j}$ is orientable, the result follows from an argument similar to the above, since the $D^{2}$-bundle $N\left(F_{1}^{j}\right) \rightarrow F_{1}^{j}$ has zero Euler number. When $F_{1}^{j}$ is non-orientable, let $p: \widetilde{F}_{1}^{j} \rightarrow F_{1}^{j}$ be the orientation double cover, $\widetilde{E}_{1}^{j} \rightarrow \widetilde{F}_{1}^{j}$ the $D^{2}$-bundle induced from $N\left(F_{1}^{j}\right) \rightarrow F_{1}^{j}$ by $p$, and $\widetilde{\phi}_{1}^{j}$ the induced stable framing on $\partial \widetilde{E}_{1}^{j}$. Then by [18, Lemma 2.3], we have

$$
p_{1}\left(\widetilde{E}_{1}^{j}, \widetilde{\phi}_{1}^{j}\right)=2 p_{1}\left(N\left(F_{1}^{j}\right), \phi_{1}^{j}\right) .
$$

On the other hand, by the proof of $(1)$, we have $p_{1}\left(\widetilde{E}_{1}^{j}, \widetilde{\phi}_{1}^{j}\right)=0$, since $\widetilde{E}_{1}^{j} \rightarrow \widetilde{F}_{1}^{j}$ is a trivial $D^{2}$-bundle. Hence $p_{1}\left(N\left(F_{1}^{j}\right), \phi_{1}^{j}\right)=0$. Since the signature of $N\left(F_{1}^{j}\right)$ vanishes, we have

$$
h\left(\phi_{1}^{j}\right)=p_{1}\left(N\left(F_{1}^{j}\right), \phi_{1}^{j}\right)-3 \sigma\left(N\left(F_{1}^{j}\right)\right)=0 .
$$

This completes the proof of Lemma 3.4.

Let us return to the proof of Theorem 3.1. Let $e_{0}^{j}$ denote the self-intersection number of $F_{0}^{j}$ in $M$, and $n_{+}$(resp. $n_{-}$) the number of $e_{0}^{j}$ 's which are positive (resp. negative). By the above lemma together with (3.2), we have

$$
h(\phi)=-F \cdot F+3\left(n_{+}-n_{-}\right) .
$$

On the other hand, by the definition of the Hirzebruch defect, we have

$$
h(\phi)=p_{1}(X, \phi)-3 \sigma(X) .
$$

Furthermore, by the Novikov additivity, we have

$$
\sigma(X)=\sigma(M)-\left(n_{+}-n_{-}\right) .
$$

Hence we have

$$
p_{1}(X, \phi)=3 \sigma(M)-F \cdot F
$$

By the Hirzebruch signature theorem, we have $3 \sigma(M)=\left\langle p_{1}(M),[M]\right\rangle$, and hence (3.1) holds. This completes the proof of Theorem 3.1 . 
Remark 3.6. In the above argument, if we are given a fold map $f: M \rightarrow \mathbf{R}^{3}$, then for $F=S(f)$ and $f_{N(F)}=f \mid N(F)$, we clearly have $p_{1}(X, \phi)=0$. Hence item (5) of Theorem 3.1 follows from (3.1).

Remark 3.7. By using a formula of Rohlin [25] and Guillou-Marin [14], Sakuma [35] has shown that for a fold map $f: M \rightarrow \mathbf{R}^{3}$ of a closed oriented 4-manifold $M$ with $H_{1}(M ; \mathbf{Z})=0$, the self-intersection number $S(f) \cdot S(f)$ must satisfy

$$
S(f) \cdot S(f) \equiv-\sigma(M)(\bmod 4) .
$$

This is nothing but the modulo four reduction of our formula of Theorem 3.1 $(5)$, since $\left\langle p_{1}(M),[M]\right\rangle=3 \sigma(M) \equiv-\sigma(M)(\bmod 4)$ by the Hirzebruch signature theorem. In fact, the formula in (5)

$$
S(f) \cdot S(f)=\left\langle p_{1}(M),[M]\right\rangle
$$

has been generalized for general dimensions in [24].

\section{Constructing a desired embedded surface}

In this section, we prove the following.

Theorem 4.1. Let $M$ be a closed connected oriented 4-manifold. Then the following four are equivalent.

(1) There exists an embedded surface $F=F_{0} \cup F_{1}$ which satisfies (1)-(5) of Theorem 3.1.

(2) The intersection form of $M$ is not isomorphic to $\pm I_{1}$ or $\pm I_{2}$, where

$$
I_{1}=(1) \quad \text { and } \quad I_{2}=\left(\begin{array}{ll}
1 & 0 \\
0 & 1
\end{array}\right) \text {. }
$$

(3) There exists a homology class $\xi \in H_{2}(M ; \mathbf{Z})$ such that $\xi \cdot \xi=\left\langle p_{1}(M),[M]\right\rangle$.

(4) There exists a cohomology class $v \in H^{2}(M ; \mathbf{Z})$ such that $v \smile v=p_{1}(M) \in$ $H^{4}(M ; \mathbf{Z})$.

Remark 4.2. It is known [22] that the intersection form of $M$ is isomorphic to $\pm I_{1}$ or $\pm I_{2}$ if and only if it is definite of rank 1 or 2 .

Proof of Theorem 4.1. (3) $\Longleftrightarrow(4)$ : This follows from the Poincaré duality.

$(1) \Longrightarrow(2)$ : Suppose that the intersection form of $M$ is isomorphic to $I_{1}=(1)$. If condition (1) is satisfied, then the homology class $\xi \in H_{2}(M ; \mathbf{Z})$ represented by $F_{0}$ satisfies $\xi \cdot \xi=\left\langle p_{1}(M),[M]\right\rangle=3 \sigma(M)=3$. Suppose that $\xi$ represents $n$ times a generator of the free part $H_{2}(M ; \mathbf{Z}) /$ Tor $H_{2}(M ; \mathbf{Z})$. Then we must have $n^{2}=3$, which has no integer solution. Thus the intersection form of $M$ is not isomorphic to $I_{1}$. By the same argument we see that $-I_{1}$ does not appear, either. When the 
intersection form of $M$ is isomorphic to $\pm I_{2}$, we must have $m^{2}+n^{2}=6$ for some integers $m$ and $n$, which is impossible again. Thus the above intersection forms do not appear, either.

$(2) \Longrightarrow(3)$ : Let us suppose that the intersection form $I_{M}$ of $M$ is not isomorphic to $\pm I_{1}$ or $\pm I_{2}$ and construct a desired homology class $\xi$.

Case 1. When $I_{M}$ is of odd type.

By the theory of unimodular bilinear forms [22] together with a theorem of Donaldson [9], $I_{M}$ is isomorphic to $m I_{1} \oplus n\left(-I_{1}\right)$ for some non-negative integers $m$ and $n$, where $k I$ denotes the direct sum of $k$ copies of a bilinear form $I$ and $m+n>0$. Let $\left\{\alpha_{1}, \ldots, \alpha_{m}, \beta_{1}, \ldots, \beta_{n}\right\}$ be a basis of $H_{2}(M ; \mathbf{Z}) /$ Tor $H_{2}(M ; \mathbf{Z})$ with respect to which $I_{M}$ is represented by $m I_{1} \oplus n\left(-I_{1}\right)$.

Lemma 4.3. If $(m, n) \neq(1,0),(0,1),(2,0),(0,2)$, then there exists an element

$$
\bar{\xi}=\sum_{i=1}^{m} a_{i} \alpha_{i}+\sum_{j=1}^{n} b_{j} \beta_{j}
$$

of $H_{2}(M ; \mathbf{Z}) /$ Tor $H_{2}(M ; \mathbf{Z})$ such that $\bar{\xi} \cdot \bar{\xi}=3(m-n)$.

Proof. We may assume that $m \geq n$. Let us list explicit solutions for certain values of $(m, n)$ as follows:

$$
\begin{aligned}
& (m, n)=(3,0):\left(a_{1}, a_{2}, a_{3}\right)=(3,0,0), \\
& (m, n)=(4,0):\left(a_{1}, a_{2}, a_{3}, a_{4}\right)=(3,1,1,1), \\
& (m, n)=(5,0):\left(a_{1}, a_{2}, a_{3}, a_{4}, a_{5}\right)=(3,2,1,1,0), \\
& (m, n)=(1,1):\left(a_{1}, b_{1}\right)=(1,1) \\
& (m, n)=(2,1):\left(a_{1}, a_{2}, b_{1}\right)=(2,0,1), \\
& (m, n)=(3,1):\left(a_{1}, a_{2}, a_{3}, b_{1}\right)=(3,1,0,2) .
\end{aligned}
$$

Then we can easily construct explicit solutions for all the remaining values of $(m, n)$ combining the above solutions.

Case 2. When $I_{M}$ is of even type.

By the theory of unimodular bilinear forms [22] together with a theorem of Donaldson [9], $I_{M}$ is the zero form or is isomorphic to $m E_{8} \oplus n U$ with $m \in \mathbf{Z}$ and $n>0$, where

$$
E_{8}=\left(\begin{array}{llllllll}
2 & 1 & 0 & 0 & 0 & 0 & 0 & 0 \\
1 & 2 & 1 & 0 & 0 & 0 & 0 & 0 \\
0 & 1 & 2 & 1 & 0 & 0 & 0 & 0 \\
0 & 0 & 1 & 2 & 1 & 0 & 0 & 0 \\
0 & 0 & 0 & 1 & 2 & 1 & 0 & 1 \\
0 & 0 & 0 & 0 & 1 & 2 & 1 & 0 \\
0 & 0 & 0 & 0 & 0 & 1 & 2 & 0 \\
0 & 0 & 0 & 0 & 1 & 0 & 0 & 2
\end{array}\right), \quad U=\left(\begin{array}{ll}
0 & 1 \\
1 & 0
\end{array}\right)
$$


Note that $E_{8}$ is unimodular of even type with signature 8 . Here, for $m<0$, $m E_{8}$ denotes the direct sum of $|m|$ copies of $-E_{8}$. Let

$$
\left\{\alpha_{11}, \ldots, \alpha_{18}, \ldots, \alpha_{m 1}, \ldots, \alpha_{m 8}, \beta_{11}, \beta_{12}, \ldots, \beta_{n 1}, \beta_{n 2}\right\}
$$

be the corresponding basis of $H_{2}(M ; \mathbf{Z}) /$ Tor $H_{2}(M ; \mathbf{Z})$. Then the following lemma can easily be checked.

Lemma 4.4. The element

$$
\bar{\xi}=\sum_{i=1}^{m}\left(2 \alpha_{i 1}+2 \alpha_{i 7}+2 \alpha_{i 8}\right)
$$

of $H_{2}(M ; \mathbf{Z}) /$ Tor $H_{2}(M ; \mathbf{Z})$ satisfies $\xi \cdot \xi=24 m$.

Hence, in both cases, an element $\xi \in H_{2}(M ; \mathbf{Z})$ representing

$$
\bar{\xi} \in H_{2}(M ; \mathbf{Z}) / \text { Tor } H_{2}(M ; \mathbf{Z})
$$

satisfies the property as in (3).

Remark 4.5. When the signature $\sigma(M)$ of $M$ is divisible by four, the solution $\xi \in H_{2}(M ; \mathbf{Z})$ can be chosen to be characteristic: i.e., we can choose $\xi$ so that its modulo two reduction coincides with the Poincaré dual of the second StiefelWhitney class $w_{2}(M) \in H^{2}(M ; \mathbf{Z} / 2 \mathbf{Z})$. This can be proved as follows.

First note that $w_{2}(M)$ is the modulo two reduction of an integral cohomology class (for example, see $[15, \S 4])$. Let $\zeta \in H_{2}(M ; \mathbf{Z})$ be its Poincaré dual. Then,

$$
\zeta \cdot \eta \equiv \eta \cdot \eta(\bmod 2)
$$

holds for all $\eta \in H_{2}(M ; \mathbf{Z})$ due to Wu's formula. On the other hand, as the proofs of Lemmas 4.3 and 4.4 show, $\bar{\xi} \in H_{2}(M ; \mathbf{Z}) /$ Tor $H_{2}(M ; \mathbf{Z})$ can be chosen so that

$$
\bar{\xi} \cdot \bar{\eta} \equiv \bar{\eta} \cdot \bar{\eta}(\bmod 2)
$$

for all $\bar{\eta} \in H_{2}(M ; \mathbf{Z}) /$ Tor $H_{2}(M ; \mathbf{Z})$, provided that $\sigma(M) \equiv 0(\bmod 4)$. This implies that $\bar{\zeta}-\bar{\xi}$ is divisible by two, where $\bar{\zeta} \in H_{2}(M ; \mathbf{Z}) /$ Tor $H_{2}(M ; \mathbf{Z})$ is the class represented by $\zeta$. Hence, there exists a representative $\xi \in H_{2}(M ; \mathbf{Z})$ of $\bar{\xi}$ such that $\zeta-\xi$ is divisible by two. Then such a homology class $\xi$ satisfies the desired properties.

$(3) \Longrightarrow(1)$ : Let $\eta \in H_{2}(M ; \mathbf{Z})$ be a homology class such that $\xi+\eta$ is characteristic. Such a homology class does exist, since the second Stiefel-Whitney class $w_{2}(M) \in H^{2}(M ; \mathbf{Z} / 2 \mathbf{Z})$ is always a modulo two reduction of some element of $H^{2}(M ; \mathbf{Z})$ as mentioned in Remark 4.5. Since $\xi \cdot(\xi+\eta) \equiv \xi \cdot \xi(\bmod 2)$, we see that $\xi \cdot \eta \equiv 0(\bmod 2)$. Let $F_{\xi}$ and $F_{\eta}$ be connected oriented surfaces embedded in $M$ which represent $\xi$ and $\eta$ respectively. Such surfaces do exist by [38]. We may assume that $F_{\xi}$ and $F_{\eta}$ are in general position and intersect at an even number of points $p_{1}, \ldots, p_{2 k}$. Let $A_{1}, \ldots, A_{k}$ be disjointly embedded arcs in $F_{\xi}$ such that $A_{i}$ 
connects $p_{2 i-1}$ and $p_{2 i}$. Let $T_{i}, i=1, \ldots, k$, be the $D^{2}$-bundle over $A_{i}$ which is the restriction of the normal disk bundle of $F_{\xi}$ embedded in $M$. We may assume that the union of the fibers over the end points of $A_{i}$ coincide with $F_{\eta} \cap T_{i}$. Let $F_{\eta}^{\prime}$ be the surface obtained by smoothing

$$
\left(F_{\eta}-\cup_{i=1}^{k}\left(F_{\eta} \cap T_{i}\right)\right) \cup\left(\cup_{i=1}^{k} C_{i}\right),
$$

where $C_{i}$ is the boundary $S^{1}$-bundle over $A_{i}$ associated with $T_{i}$. Note that $F_{\eta}^{\prime}$ is an embedded surface which may possibly be non-orientable such that $F_{\xi} \cap F_{\eta}^{\prime}=\emptyset$ and $F_{\eta}^{\prime}$ represents the modulo two reduction of $\eta$ in $H_{2}(M ; \mathbf{Z} / 2 \mathbf{Z})$. In particular, $F_{\xi} \cup F_{\eta}^{\prime}$ is characteristic.

Furthermore, since $(\xi+\eta) \cdot(\xi+\eta) \equiv \sigma(M)(\bmod 8)($ see $[22])$ and $\xi \cdot \xi=3 \sigma(M)$ by our assumption, we have $\eta \cdot \eta \equiv 0(\bmod 2)$. Hence $F_{\eta}^{\prime} \cdot F_{\eta}^{\prime}$ is even. Then by taking the connected sum of $F_{\eta}^{\prime}$ with a suitable closed connected non-orientable surface embedded in $D^{4} \subset M$, we may assume that $F_{\eta}^{\prime} \cdot F_{\eta}^{\prime}=0$.

Lemma 4.6. We have $\chi(M)-\left(\chi\left(F_{\xi}\right)-\chi\left(F_{\eta}^{\prime}\right)\right) \equiv 0(\bmod 2)$.

Proof. Since $F_{\xi} \cup F_{\eta}^{\prime} \subset M$ is characteristic, we have

$$
\left(F_{\xi} \cup F_{\eta}^{\prime}\right) \cdot\left(F_{\xi} \cup F_{\eta}^{\prime}\right)+2 \chi\left(F_{\xi} \cup F_{\eta}^{\prime}\right) \equiv \sigma(M)(\bmod 4),
$$

provided that $H_{1}(M ; \mathbf{Z})=0($ see $[25,14,21,42])$. If $H_{1}(M ; \mathbf{Z})$ does not vanish, then we can perform spin surgeries along embedded circles in the spin 4-manifold $M \backslash\left(F_{\xi} \cup F_{\eta}^{\prime}\right)$ to obtain a closed oriented 4-manifold $M^{\prime}$ with $H_{1}\left(M^{\prime} ; \mathbf{Z}\right)=0$ such that $F_{\xi} \cup F_{\eta}^{\prime}$ is characteristic. Hence (4.1) holds even if $H_{1}(M ; \mathbf{Z}) \neq 0$, since $\sigma(M)=\sigma\left(M^{\prime}\right)$. Finally, since $\chi(M) \equiv \sigma(M)(\bmod 2)$ and $\left(F_{\xi} \cup F_{\eta}^{\prime}\right) \cdot\left(F_{\xi} \cup F_{\eta}^{\prime}\right)=$ $3 \sigma(M)$, we have the result.

By taking the connected sum of $F_{\xi}$ or $F_{\eta}^{\prime}$ with a closed connected oriented surface of an appropriate genus embedded in $D^{4} \subset M$, we may assume that $\chi(M)=\chi\left(F_{\xi}\right)-\chi\left(F_{\eta}^{\prime}\right)$ due to the above lemma. Thus $F=F_{0} \cup F_{1}$ with $F_{0}=F_{\xi}$ and $F_{1}=F_{\eta}^{\prime}$ satisfies the properties (1)-(5) of Theorem 3.1. This completes the proof of Theorem 4.1.

Remark 4.7. As the above proof shows, if condition (3) of Theorem 4.1 holds, then $F_{0}$ and $F_{1}$ in $(1)$ can be chosen to be connected.

Note that if a given fold map $f$ satisfies $S_{0}(f)=\emptyset$ or $S_{1}(f)=\emptyset$, then we can easily modify it homotopically to obtain another fold map whose corresponding sets are nonempty. Therefore, combining Theorems 3.1 and 4.1, we obtain Theorem 1.1 in $\S 1$.

Remark 4.8. (1) In [26], the author showed that if a closed orientable 4-manifold has the integral homology of $\mathbf{C} P^{2}$, then it cannot admit a fold map into $\mathbf{R}^{3}$, by using Sakuma's result mentioned in Remark 3.7 (for another proof, see [2]). 
This result was extended to 4 -manifolds $M$ with $\operatorname{dim}_{\mathbf{Z} / 2 \mathbf{Z}} H_{2}(M ; \mathbf{Z} / 2 \mathbf{Z})=1$ by Sakuma [37], who used Yamada's quadratic form on 4-manifolds [42]. Note that $\operatorname{dim}_{\mathbf{Z} / 2 \mathbf{Z}} H_{2}(M ; \mathbf{Z} / 2 \mathbf{Z})=1$ if and only if $H_{2}(M ; \mathbf{Z})$ is isomorphic to the direct sum of $\mathbf{Z}$ and an odd torsion by the universal coefficient theorem. Thus, our result is a generalization of these two, and gives a complete answer to the existence problem of fold maps on closed orientable 4-manifolds. (Note that Sakuma [36] had conjectured that closed orientable 4-manifolds of odd Euler characteristics cannot admit a fold map into $\mathbf{R}^{3}$ (see also [16, 34]). Our result shows that the conjecture is false.)

(2) In [24], it has been shown that the self-intersection class of the singular set - which can be considered as a variant of a Thom polynomial - coincides with $p_{1}(M)$ for a fold map $M \rightarrow \mathbf{R}^{3}$ of a closed orientable 4-manifold $M$. (As has been seen above, this also follows from our proof of Theorem 3.1.) Hence, Theorem 1.1 can be interpreted as follows: there exists a fold map $M \rightarrow \mathbf{R}^{3}$ if and only if there are no obstructions coming from the Thom polynomials. Compare this with the result obtained in [32] about stable maps between 4-manifolds (see also Remark 4.13 below).

(3) When the result mentioned above for homology $\mathbf{C} P^{2}$ was obtained in [26], the author had the impression that such a result should be peculiar to 4dimensions, since we used a variant of the Rohlin theorem, which is peculiar to 4-dimensions. However, according to [24], this is not true. In fact, in [24], similar non-existence theorems are obtained for other dimensions as well.

As immediate corollaries to our main theorem, we have the following.

Corollary 4.9. Let $M_{1}$ and $M_{2}$ be closed connected orientable 4-manifolds which are homotopy equivalent to each other. Then, $M_{1}$ admits a fold map into $\mathbf{R}^{3}$ if and only if $M_{2}$ does.

Note that the above result does not hold for fold maps with restricted indices, namely for special generic maps. For details, see [27, 29, 31, 33].

Corollary 4.10. Let $M$ be a closed connected oriented 4-manifold.

(1) There always exists a closed oriented 4-manifold which is oriented cobordant to $M$ and which admits a fold map into $\mathbf{R}^{3}$.

(2) If $|\sigma(M)| \neq 1,2$, then every closed oriented 4-manifold that is oriented cobordant to $M$ admits a fold map into $\mathbf{R}^{3}$.

Note that Corollary 4.10 (1) follows also from the example constructed in [30] mentioned below. According to [24], examples as in Corollary 4.10 (2) do not exist for general dimensions.

Remark 4.11. For explicit examples of fold maps, see [26, 30]. For example, an explicit example of a fold map $\mathbf{C} P^{2} \sharp 2 \overline{\mathbf{C} P^{2}} \rightarrow \mathbf{R}^{3}$ is constructed in [30], where 
$\mathbf{C} P^{2} \sharp 2 \overline{\mathbf{C} P^{2}}$ denotes the connected sum of the complex projective plane $\mathbf{C} P^{2}$ and two copies of $\mathbf{C} P^{2}$ with orientation reversed.

Remark 4.12. The three conditions (1)-(3) of Theorem 1.1 are all equivalent to the following.

(4) For every continuous map $g: M \rightarrow N$ into an orientable 3 -manifold $N$, there exists a fold map of $M$ into $N$ homotopic to $g$.

(5) There exists a fold map $f: M \rightarrow N$ for some orientable 3-manifold $N$.

This can be proved by using the fact that $N$ is parallelizable and that every continuous map of a closed surface into $N$ is homotopic to an immersion [41].

Remark 4.13. Let $f: M \rightarrow N$ be a $C^{\infty}$ stable map of a closed orientable 4manifold into an orientable 3 -manifold. It is well-known that the possible singularities of $f$ are all Morin singularities; namely, folds, cusps and swallowtails. As has been shown in [3], swallowtails, which are zero dimensional singularities, can always be eliminated by homotopy. By Remark 4.12, cusps, which are 1dimensional singularities, can be eliminated by homotopy if and only if $M$ satisfies one of the three conditions of Theorem 1.1. Note that the primary obstruction to the elimination of cusps — the Thom polynomial for cusps — always vanishes (see $[36])$.

\section{Stable span and fold maps}

In this section, we discuss the relationship between the fold map problem and the vector field problem. Let us begin by recalling the following definition.

Definition 5.1. For a smooth manifold $M$, the maximal number of everywhere linearly independent vector fields of $M$ is called the span of $M$ and is denoted by span $M$. Furthermore, the maximal number of everywhere linearly independent sections of $T M \oplus \varepsilon^{1}$ subtracted by one is called the stable span of $M$ and is denoted by $\operatorname{span}^{0} M$, where $\varepsilon^{1}$ denotes the trivial line bundle over $M$ (for details, see [19], for example).

Ando $[4,5,6]$ has shown the following theorem by using the homotopy principle for fold maps in the 2-jet level (see [3, Theorem 1]).

Theorem 5.2. Let $M$ be a smooth $n$-dimensional manifold. If there exists a fiberwise epimorphism $T M \oplus \varepsilon^{1} \rightarrow \varepsilon^{p}$ for some $p$ with $n \geq p \geq 2$, then there exists a fold map $f: M \rightarrow \mathbf{R}^{p}$, where $\varepsilon^{p}$ denotes the trivial p-plane bundle over $M$. If $n-p+1$ is odd, then the converse also holds.

Remark 5.3. In fact, Ando [6] has proved that there exists a fiberwise epimorphism $T M \oplus \varepsilon^{1} \rightarrow \varepsilon^{p}$ if and only if there exists a fold map $f: M \rightarrow \mathbf{R}^{p}$ such that 
the codimension one immersion $f \mid S(f): S(f) \rightarrow \mathbf{R}^{p}$ has trivial normal bundle (or equivalently, $S(f)$ is orientable). Recall that if $n-p+1$ is odd, then for a fold map $f$ of an $n$-dimensional manifold into $\mathbf{R}^{p}$, the normal bundle of the immersion $f \mid S(f)$ is always trivial (for example, see [26, Lemma 2.2]).

It is easy to see that there exists a fiberwise epimorphism $T M \oplus \varepsilon^{1} \rightarrow \varepsilon^{p}$ if and only if $\operatorname{span}^{0} M \geq p-1$. Hence, we have the following.

Corollary 5.4. For a smooth $n$-dimensional manifold $M$ and an integer $p$ with $n \geq p \geq 1$, we have the following.

(1) We have $\operatorname{span}^{0} M \geq p-1$ if and only if there exists a fold map $f: M \rightarrow \mathbf{R}^{p}$ such that the normal bundle of the immersion $f \mid S(f): S(f) \rightarrow \mathbf{R}^{p}$ is trivial.

(2) When $n-p+1$ is odd, $\operatorname{span}^{0} M \geq p-1$ if and only if there exists a fold $\operatorname{map} f: M \rightarrow \mathbf{R}^{p}$.

It has been known that the stable span of 4-manifolds satisfies the following (for details, see $[40,19]$ ).

Proposition 5.5. Let $M$ be a closed connected oriented 4-manifold.

(1) We have $\operatorname{span}^{0} M \geq 1$ if and only if $\chi(M)$ is even.

(2) We have $\operatorname{span}^{0} M \geq 2$ if and only if there exists a characteristic homology class $\xi \in H_{2}(M ; \mathbf{Z})$ with $\xi \cdot \xi=\left\langle p_{1}(M),[M]\right\rangle$.

(3) We have $\operatorname{span}^{0} M \geq 3$ if and only if $M$ is spin and $p_{1}(M)$ vanishes.

Remark 5.6. In terms of the signature of $M$, the first two conditions of Proposition 5.5 can be interpreted as follows.

(1) The Euler characteristic $\chi(M)$ is even if and only if the signature $\sigma(M)$ is even.

(2) There exists a characteristic homology class $\xi \in H_{2}(M ; \mathbf{Z})$ with $\xi \cdot \xi=$ $\left\langle p_{1}(M),[M]\right\rangle$ if and only if $\sigma(M) \equiv 0(\bmod 4)$.

Item (1) follows easily from the definitions of $\chi(M)$ and $\sigma(M)$. For (2), first note that if such a characteristic homology class $\xi$ exists, then

$$
3 \sigma(M)=\left\langle p_{1}(M),[M]\right\rangle=\xi \cdot \xi \equiv \sigma(M)(\bmod 8),
$$

and hence $\sigma(M) \equiv 0(\bmod 4)$. Conversely, if $\sigma(M) \equiv 0(\bmod 4)$, then by the proofs of Lemmas 4.3 and 4.4 , we can construct a homology class $\xi$ with $\xi \cdot \xi=$ $\left\langle p_{1}(M),[M]\right\rangle$ such that $\xi$ is characteristic as has been noted in Remark 4.5.

By the above results, we have the following.

Proposition 5.7. Let $M$ be a closed connected oriented 4-manifold. Then the following five conditions are equivalent. 
(1) There exists a fold map $f: M \rightarrow \mathbf{R}^{3}$ such that the codimension one immersion $f \mid S(f): S(f) \rightarrow \mathbf{R}^{3}$ has trivial normal bundle (or equivalently, $S(f)$ is orientable).

(2) $\operatorname{span}^{0} M \geq 2$.

(3) There exists a characteristic homology class $\xi \in H_{2}(M ; \mathbf{Z})$ with $\xi \cdot \xi=$ $\left\langle p_{1}(M),[M]\right\rangle$.

(4) There exists a cohomology class $v \in H^{2}(M ; \mathbf{Z})$ such that its modulo two reduction coincides with $w_{2}(M) \in H^{2}(M ; \mathbf{Z} / 2 \mathbf{Z})$ and that $v \smile v=p_{1}(M) \in$ $H^{4}(M ; \mathbf{Z})$.

(5) $\sigma(M) \equiv 0(\bmod 4)$.

In fact, we can prove the equivalence $(1) \Longleftrightarrow(3)$ of Proposition 5.7 by using our Theorem 1.1 and its proof as follows. Suppose that (1) holds. Then by Theorem 3.1, the homology class $\xi \in H_{2}(M ; \mathbf{Z})$ represented by $S(f)$ satisfies the desired property in (3). Conversely, if (3) holds, then the homology class $\eta$ in the proof of Theorem 4.1 can be chosen to be zero. Then, the surface $F_{\eta}^{\prime}$ is orientable and (1) holds.

Remark 5.8. In Proposition 5.7, items (1)-(5) are all equivalent to the following.

(6) There exists a Morin map $f: M \rightarrow \mathbf{R}^{3}$ whose cokernel bundle over $S(f)$ is trivial.

Recall that a smooth map is a Morin map if it has only Morin singularities. For Morin maps and their cokernel bundles, see [23, 13, 12, 28], for example. The above assertion can be proved by generalizing [26, Lemma 3.1] to Morin maps with trivial cokernel bundles, which is easy.

Remark 5.9. Let $M$ be a closed oriented 4-manifold. Clearly, if $\operatorname{span}^{0} M \geq 3$, then $\operatorname{span}^{0} M \geq 2$. However, even if there exists a fold map $f: M \rightarrow \mathbf{R}^{3}$, there may not exist a fold map of $M$ into $\mathbf{R}^{2}$. For example, any closed connected oriented 4-manifold whose second Betti number is odd and is greater than or equal to three admits a fold map into $\mathbf{R}^{3}$, but not into $\mathbf{R}^{2}$.

Remark 5.10. For a closed $n$-dimensional manifold $M$ with $n \geq 2$, the following three are equivalent to each other.

(1) $\operatorname{span}^{0} M \geq 1$.

(2) $\chi(M) \equiv 0(\bmod 2)$.

(3) There exists a fold map $M \rightarrow \mathbf{R}^{2}$.

We can prove the equivalence from a singularity theoretical viewpoint as follows. Item (3) implies (1), since the singular set of any fold map $M \rightarrow \mathbf{R}^{2}$ is 1-dimensional and is orientable (for details, see [26, Lemma 3.1]). Item (1) implies (2) by an easy argument of characteristic classes. Finally, (2) implies (3) by Levine's cusp elimination theorem [20]. 
Remark 5.11. Adachi [1] has shown that for an open orientable 4-manifold $M$, there exists a submersion $M \rightarrow \mathbf{R}^{2}$ if and only if there exists a characteristic cohomology class $v \in H^{2}(M ; \mathbf{Z})$ such that $v \smile v=p_{1}(M)=0$.

Adachi [1] has also shown that a closed connected orientable 4-manifold $M$ immerses into $\mathbf{R}^{6}$ if and only if there exists a characteristic cohomology class $v \in H_{2}(M ; \mathbf{Z})$ such that $v \smile v=-p_{1}(M)$. (According to [7], this is equivalent to that $\chi(M)$ is even and the intersection form $I_{M}$ is either the zero form or is indefinite.) See also [17].

Compare these results with ours.

\section{References}

[1] M. Adachi, A remark on submersions and immersions with codimension one or two, J. Math. Kyoto Univ. 9 (1969), 393-404.

[2] P. M. Akhmetiev and R. R. Sadykov, A remark on elimination of singularities for mappings of 4-manifolds into 3-manifolds, to appear in Topology Appl.

[3] Y. Ando, On the elimination of Morin singularities, J. Math. Soc. Japan 37 (1985), 471487; Erratum 39 (1987), 537.

[4] Y. Ando, Folding maps and the surgery theory on manifolds, J. Math. Soc. Japan 53 (2001), $357-382$.

[5] Y. Ando, Fold-maps and the space of base point preserving maps of spheres, J. Math. Kyoto Univ. 41 (2002), 693-737.

[6] Y. Ando, Existence theorems of fold-maps, preprint.

[7] C. Biasi and J. A. Daccach, Imersões de variedades de dimensão 4 em $\mathbf{R}^{6}$ (in Portuguese), preprint.

[8] V. M. Buchstaber and K. E. Feldman, The index of an equivariant vector field and addition theorems for Pontryagin classes, Izv. Math. 64 (2000), 223-247.

[9] S. K. Donaldson, The orientation of Yang-Mills moduli spaces and 4-manifold topology, J. Diff. Geom. 26 (1987), 397-428.

[10] J. M. Èliašberg, On singularities of folding type, Math. USSR-Izv. 4 (1970), 1119-1134.

[11] J. M. Ėliašberg, Surgery of singularities of smooth mappings, Math. USSR-Izv. 6 (1972), 1302-1326.

[12] T. Fukuda, Topology of folds, cusps and Morin singularities, in: A Fete of Topology, eds. Y. Matsumoto, T. Mizutani and S. Morita, 331-353, Academic Press, 1987.

[13] M. Golubitsky and V. Guillemin, Stable mappings and their singularities, Grad. Texts in Math. 14, Springer, New York, Heidelberg, Berlin, 1973.

[14] L. Guillou and A. Marin, Une extension d'un théorème de Rohlin sur la signature, $C$. $R$. Acad. Sci. Paris 285 (1977), 95-98.

[15] F. Hirzebruch and H. Hopf, Felder von Flächenelementen in 4-dimensionalen Mannigfaltigkeiten, Math. Ann. 136 (1958), 156-172.

[16] S. Kikuchi and O. Saeki, Remarks on the topology of folds, Proc. Amer. Math. Soc. 123 (1995), 905-908.

[17] R. Kirby, The topology of 4-manifolds, Lecture Notes in Math. 1374, Springer-Verlag, 1989.

[18] R. Kirby and P. Melvin, Canonical framings for 3-manifolds, Proc. of 6th Gökova GeometryTopology Conference, Turkish J. Math. 23 (1999), 89-115.

[19] J. Korbaš and P. Zvengrowski, The vector field problem: a survey with emphasis on specific manifolds, Expos. Math. 12 (1994), 3-30.

[20] H. Levine, Elimination of cusps, Topology 3 (suppl. 2) (1965), 263-296. 
[21] Y. Matsumoto, An elementary proof of Rochlin's signature theorem and its extension by Guillou and Marin, in: À la recherche de la topologie perdue, 119-139, Progr. Math., Vol. 62, Birkhäuser, Boston, 1986.

[22] J. Milnor and D. Husemoller, Symmetric bilinear forms, Ergebnisse Math., Band 73, Springer, Berlin, Heidelberg, New York, 1973.

[23] B. Morin, Formes canoniques des singularités d'une application différentiable, C. R. Acad. Sci. Paris 260 (1965), 5662-5665, 6503-6506.

[24] T. Ohmoto, O. Saeki, and K. Sakuma, Self-intersection class for singularities and its application to fold maps, preprint.

[25] V. A. Rohlin, Proof of Gudkov's hypothesis, Funct. Anal. Appl. 6 (1972), 136-138.

[26] O. Saeki, Notes on the topology of folds, J. Math. Soc. Japan 44 (1992), 551-566.

[27] O. Saeki, Topology of special generic maps into $\mathbf{R}^{3}$, in: Workshop on Real and Complex Singularities, Matemática Contemporânea 5 (1993), 161-186.

[28] O. Saeki, Studying the topology of Morin singularities from a global viewpoint, Math. Proc. Camb. Phil. Soc. 117 (1995), 223-235.

[29] O. Saeki, Global theory of singularities of differentiable mappings: present status and prospects (translation of Sūgaku 48 (1996), 385-399), Sugaku Expositions 12 (1999), 21-37.

[30] O. Saeki, Topology of singular fibers of stable maps, preprint.

[31] O. Saeki and K. Sakuma, On special generic maps into $\mathbf{R}^{3}$, Pacific J. Math. 184 (1998), $175-193$.

[32] O. Saeki and K. Sakuma, Stable maps between 4-manifolds and elimination of their singularities, J. London Math. Soc. (2) 59 (1999), 1117-1133.

[33] O. Saeki and K. Sakuma, Special generic maps of 4-manifolds and compact complex analytic surfaces, Math. Ann. 313 (1999), 617-633.

[34] O. Saeki and K. Sakuma, Elimination of singularities: Thom polynomial and beyond, in: Singularity Theory, ed. Bill Bruce and David Mond, Proceedings of the European Singularities Conference, Liverpool, August 1996, Dedicated to C. T. C. Wall on the occasion of his 60th birthday, 291-304, London Math. Soc. Lect. Note Series 263, Cambridge Univ. Press, 1999.

[35] K. Sakuma, On special generic maps of simply connected $2 n$-manifolds into $\mathbf{R}^{3}$, Topology Appl. 50 (1993), 249-261.

[36] K. Sakuma, On the topology of simple fold maps, Tokyo J. Math. 17 (1994), 21-31.

[37] K. Sakuma, A note on nonremovable cusp singularities, Hiroshima Math. J. 31 (2001), 461-465.

[38] R. Thom, Quelques propriétés globales des variétés différentiables, Comment. Math. Helv. 28 (1954), 17-86.

[39] R. Thom, Les singularités des applications différentiables, Ann. Inst. Fourier (Grenoble) 6 (1955-56), 43-87.

[40] E. Thomas, Vector fields on low dimensional manifolds, Math. Z. 103 (1968), 85-93.

[41] H. Whitney, The singularities of a smooth $n$-manifold in $(2 n-1)$-space, Ann. of Math. (2) 45 (1944), 247-293.

[42] Y. Yamada, An extension of Whitney's congruence, Osaka J. Math. 32 (1995), 185-192.

O. Saeki

Faculty of Mathematics

Kyushu University

Hakozaki

Fukuoka 812-8581

Japan

e-mail: saeki@math.kyushu-u.ac.jp

(Received: May 7, 2002) 